\title{
Fulranumab in patients with interstitial cystitis/bladder pain syndrome: observations from a randomized, double- blind, placebo-controlled study
}

Hao Wang ${ }^{1}$, Lucille J. Russell ${ }^{2}$, Kathleen M. Kelly², Steven Wang ${ }^{2}$ and John Thipphawong ${ }^{2^{*}}$

\begin{abstract}
Background: This study was designed to evaluate the efficacy and safety of fulranumab, a fully human monoclonal antibody directed against nerve growth factor (NGF), for pain relief in patients with interstitial cystitis/bladder pain syndrome (IC/BPS).

Methods: In this multicenter, double-blind study, adults with IC/BPS (i.e., interstitial cystitis symptom index [ICSI] total score $\geq 8$ ) accompanied by chronic, moderate-to-severe pain were randomized to fulranumab $9 \mathrm{mg}$ or matching placebo, administered subcutaneously at weeks 1, 5, and 9. The primary efficacy endpoint was change from baseline to study endpoint (week 12 or at withdrawal) in average daily pain intensity score. Key secondary endpoints included change from baseline to study endpoint in worst pain intensity score, ICSI total score, Pelvic Pain and Urgency/Frequency total score, Patient Perception of Bladder Condition score, and global response assessment.
\end{abstract}

Results: This study was terminated prematurely based on concern that this class may be associated with rapidly progressing osteoarthritis or osteonecrosis. Thirty-one patients (of the targeted 70 patients) were randomized, 17 to placebo and 14 to fulranumab, with 15 and 10 patients, respectively, receiving all 3 doses of double-blind treatment. In ANOVA analyses, there was no statistically significant difference between treatment groups for the primary endpoint (LS mean difference $[95 \% \mathrm{CI}]$ vs. placebo, $-0.2[-1.52,1.10]$ ) or any of the secondary endpoints. Fulranumab was well tolerated, with no patient discontinuing due to an adverse event or experiencing a jointrelated serious adverse event over a 26-week follow-up period. No events related to the neurologic or motor systems were reported.

Conclusions: Efficacy was not demonstrated in the present study with the single dose tested and a limited sample size, leading to lack of statistical power. These findings do not exclude the possibility that fulranumab would provide clinical benefit in a larger study and/or specific populations (phenotypes) in this difficult to treat pain condition.

Trial registration: NCT01060254, registered January 29, 2010.

Keywords: Fulranumab, Pain, Analgesia, Interstitial cystitis, Bladder

* Correspondence: JThippha@its.jnj.com

2Janssen Research \& Development, LLC, Raritan/Titusville, NJ, USA

Full list of author information is available at the end of the article 


\section{Background}

Pain (suprapubic related to bladder filling, pelvic, and/or in extragenital locations such as the lower abdomen/back) is the hallmark symptom of interstitial cystitis/bladder pain syndrome (IC/BPS) [1]. Several lines of evidence suggest that IC/BPS can be conceptualized as a bladder pain syndrome associated with not only chronic pain, but also voiding-related symptoms and, in many, other chronic systemic pain disorders. As such, pain management is central to multimodal therapeutic strategies for IC/BPS, given its bearing on psychosocial functioning and quality of life [2, 3]. In fact, recently published IC/BPS guidelines from the American Urological Association recommend pain management as first-line treatment, which should be offered to all patients [1]. Despite the need of IC/BPS patients for analgesia, effective pharmacological treatment for their chronic pain remains elusive [4-6].

In the midst of a growing need for alternative analgesics, nerve growth factor (NGF) antagonism is a focus of drug research and development, with anti-NGF agents in the spotlight [7]. Experimental and clinical evidence has established the major role of NGF in the generation and maintenance of pain states, and specifically in bladder pain in animal models [7-9]. Several humanized and fully human anti-NGF monoclonal antibodies have entered clinical trials as potential analgesics, with preliminary results showing significant improvement in chronic pain from osteoarthritis [10] and diabetic peripheral neuropathy $[11,12]$ and mixed results for relief of chronic low back pain [13]. Efficacy for interstitial cystitis was also tested initially with positive results [14], but failed to show treatment effect versus placebo in a second study of chronic prostatitis/chronic pelvic pain syndrome [15].

Fulranumab, a fully human recombinant monoclonal antibody (immunoglobulin G), is a potent inhibitor of human NGF. Results from phase 2, randomized, placebocontrolled studies of fulranumab demonstrate a positive dose response in diabetic peripheral neuropathic pain [11], mixed efficacy results versus placebo and statistically significant improvement versus an opioid in pain of osteoarthritis [16, 17], and did not separate from placebo for low-back pain [18]. We herein report the results of a phase 2a study conducted to explore the efficacy and safety profile of fulranumab, as compared to placebo, in patients with moderate-to-severe chronic bladder pain from IC/BPS. On December 23, 2010, the United States Food and Drug Administration (FDA) placed ongoing fulranumab studies on clinical hold because of a concern that the entire class of anti-NGF antibodies may be associated with a condition representing either rapidly progressing osteoarthritis or osteonecrosis [19]. As a result, the sponsor discontinued this study prematurely, after having enrolled only 31 of the targeted 70 patients.

\section{Methods}

\section{Ethical practices}

An Independent Review Board (US) or Research Ethics Board (Canada) at each study site approved the study protocol and protocol amendments. The study was conducted in accordance with the ethical principles that have their origin in the Declaration of Helsinki, consistent with Good Clinical Practices and applicable regulatory requirements. All patients provided written informed consent before study participation. The study is registered at clinicaltrials.gov, NCT 01060254.

\section{Patients}

Study participants were adult ( $\geq 18$ to 80 years, inclusive) men and women with IC/BPS based on a total score $\geq 8$ on the validated O'Leary-Sant interstitial cystitis symptom index (ICSI) $[20,21]$ and chronic bladder pain for at least 6 months prior to screening, accompanied by urinary urgency, urinary frequency ( $\geq 8$ voids daily), and/ or nocturia. On the basis of patients' self-assessment of pain using a 11-point numerical rating scale (NRS) (0 = "no pain", $10=$ "worst pain imaginable"), the mean of the average pain intensity scores for the last 7 days of screening had to be $\geq 5$ based on at least 6 of 7 days for eligible patients [22]. Other key eligibility criteria required that patients had no evidence of a urinary tract infection or significant urological disease, including neurogenic bladder and diabetic cystopathy, and had not received intravesical therapy or undergone cystoscopy during the 6 weeks prior to screening. In addition, they had not received opioid analgesic in a dosage of oral morphine equivalent $\geq 40 \mathrm{mg} /$ day or changed drugs known to affect IC/BPS-associated pain (i.e., antidepressants, antihistamines, antispasmodics, anticholinergics, anticonvulsants) within the 4 weeks before screening.

\section{Study design}

This randomized, double-blind, placebo-controlled, multicenter study was conducted between March 2010 and June 2011 (last study visit) at 12 study sites (10 in US and 2 in Canada). Reports of spontaneous reporting of joint replacements were collected through November 2011 (explanation provided under Safety Assessments). The study comprised a screening period of up to 4 weeks, a 12-week double-blind treatment period, and a post-treatment period that concluded 26 weeks after the final dose of study drug was administered.

Eligible patients were randomized in a 1:1 ratio, based on a computer-generated randomization schedule, to fulranumab $9 \mathrm{mg}$ or matching placebo injected subcutaneously (SC) into the thigh (or abdominal wall, only if the thigh was not feasible) once every 4 weeks (i.e., at weeks 1, 5, and 9 visits). Randomization was balanced using randomly permuted blocks and was stratified by 
the presence versus absence of glomerulations and/or Hunner's ulcer on cystoscopy [23] and by baseline body weight ( $<85 \mathrm{~kg}$ vs. $\geq 85 \mathrm{~kg}$ ). Patients were followed during the double-blind treatment period at clinic visits conducted at baseline and every other week through week 13.

If patients used regularly scheduled bladder pain medications within 3 months before screening, they could continue taking them (with no dose changes) concomitantly with study drug. In addition, rescue use of acetaminophen (up to $3000 \mathrm{mg} /$ day) was allowed throughout the study.

\section{Efficacy assessments}

At each study visit, patients received a diary into which they recorded a bladder pain NRS score for average and worst pain over the previous $24 \mathrm{~h}$, urinary frequency (daytime and nocturnal), and interference with sleep for the 7 days before clinic visits. At all clinic visits, patients rated the degree to which their IC/BPS caused them problems using the Patient Perception of Bladder Condition (PPBC) instrument [24] and the number of nocturnal awakenings for voiding, pain with sexual activity, bladder/pelvic pain, and the frequency of bother with these symptoms using the Pelvic Pain and Urgency/Frequency Questionnaire (PUF) [25]. At selected clinic visits (weeks 5,9 , and 13 ), patients also rated the presence and extent of their IC/BPS symptoms (urinary urgency, urinary frequency, nocturia, pain/burning in the bladder) by completing an ICSI questionnaire and their overall status since beginning study medication by completing the 7-point global response assessment (GRA): very much worse; much worse; minimally worse; not changed; minimally improved; much improved; and very much improved.

\section{Safety assessments}

Investigators monitored adverse events throughout the study. They also performed other standard safety assessments (i.e., clinical laboratory tests, vital signs, physical examination) at prespecified time points.

An independent data monitoring committee, including 3 medical experts in pain and neurology and 1 statistician, reviewed unblinded safety data on an ongoing basis to ensure patient safety. This review did not identify any safety signal that met prespecified stopping rules.

In response to an FDA request, additional information to assess patient's joints was collected to evaluate whether rapidly progressing osteoarthritis or osteonecrosis occurred during the study. The tests included imaging data and/or historical data (e.g., X-rays, MRIs, ultrasounds, historical data pertaining to the joint replacement) of any joint that had been replaced or in which a relevant joint-related adverse event occurred.
The study remained open until November 2011 to accommodate this request.

\section{Data analysis}

Efficacy analyses were performed on the intent-to-treat (ITT) data set, namely, all patients who were randomized, received at least 1 dose of study drug, and had at least 1 efficacy evaluation during the double-blind treatment period.

\section{Efficacy endpoints and analyses}

The primary efficacy endpoint, defined as change in average pain intensity from the baseline bladder pain intensity score at study endpoint (i.e., end of the doubleblind treatment phase or at the withdrawal visit), was analyzed using an analysis of variance (ANOVA) model, which included treatment group as factor. For patients who terminated before completing 12 weeks of treatment, the last observed data were carried forward to endpoint for analysis. Analyses of secondary efficacy endpoints were conducted using a similar ANOVA model.

\section{Sample size determination}

In the absence of data on bladder pain effect size in IC/ BPS patients and specific effects of fulranumab, sample size assumptions for this study were based on those from another study [16] that was ongoing at the time the protocol for this study was written. Assuming a treatment difference of 1.4 in the change from baseline in average pain intensity score between fulranumab and placebo, a standard deviation (SD) of 2.4, and an overall discontinuation rate of $20 \%$, then a sample size of $35 \mathrm{pa}-$ tients per treatment group or total 70 patients in the entire study would have provided $78.8 \%$ power using a type 1 error rate of 0.1 and a 1 -sided 2 sample $t$-test.

\section{Results \\ Patients}

A total of 83 patients were screened and 31 eligible patients were randomized, 17 to placebo and 14 to fulranumab. On December 23, 2010, the FDA placed ongoing fulranumab studies on clinical hold because of a concern that the entire class of anti-NGF antibodies may be associated with a condition representing either rapidly progressing osteoarthritis or osteonecrosis [19]. As a result, the sponsor discontinued this study prematurely, after having enrolled only 31 of the targeted 70 patients.

Of the 31 randomized patients, 24 completed the study and 7 were withdrawn from the study prematurely (Table 1). Two of 17 patients in the placebo group and 4 of 14 patients in the fulranumab group did not receive all 3 doses of double-blind study drug. 
Table 1 Patient disposition

\begin{tabular}{lcc}
\hline & \multicolumn{2}{c}{ Number (\%) of patients } \\
\cline { 2 - 3 } & Placebo & Fulranumab 9 mg \\
\hline Randomized & $17(100)$ & $14(100)$ \\
Received all 3 doses of study drug & $15(88)$ & $10(71)$ \\
Completed the study & $14(82)$ & $10(71)$ \\
Withdrew from the study & $3(18)$ & $4(29)$ \\
Sponsor discontinued the study & $1(6)$ & $0(0)$ \\
Patient withdrew consent & $2(12)$ & $0(0)$ \\
Other & $0(0)$ & $4^{\mathrm{a}}(29)$ \\
\hline
\end{tabular}

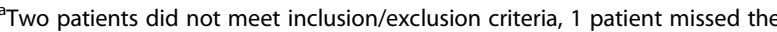

Week 9 dose, and 1 patient withdrew due to the clinical hold

The treatment groups were well-matched based on demographic and most other baseline characteristics (Table 2). The ITT data set was composed primarily of women (83.9\%); the mean (SD) age was 48.2 (12.34) years. Patients in the placebo group reported longer IC/BPS disease duration (mean, 9.4 vs. 5.8 years for the fulranumab group) and higher daytime urinary frequency (mean, 16.5 vs. 10.8); mean baseline daily NRS scores for average (6.6 vs. 5.7$)$ and worst (7.6 vs. 6.6) bladder pain scores were similar between the treatment groups, as was the frequency of nocturnal urinary frequency ( 4.9 vs. 3.7). Of the UPOINT domains, the fulranumab group had a higher percentage of patients in the infection and neurological/ systemic domains than the placebo group (Table 2).

Sixteen patients in the placebo group and 12 of 14 $(86 \%)$ in the fulranumab group received concomitant IC/BPS medications with study drug, the most common being pentosan polysulfate sodium (PPS) $(7$ patients in each treatment group); a benzodiazepine ( 6 and 5 patients in the placebo and fulranumab groups, respectively), a corticosteroid ( 4 and 2 patients in the respective groups), an antidepressant ( 7 and 3 patients in the respective groups), an antihistamine (5 and 6 patients in the respective groups), an anticholinergic (2 and 3 patients in the respective groups), an antispasmodic (1 and 3 patients in the respective groups), and an anti-infective agent ( 3 and 2 patients in the respective groups).

\section{Efficacy results}

There was no statistically significant difference between fulranumab and placebo for the primary efficacy endpoint (Table 3). The responder rates based on 30 and $50 \%$ improvement at the study endpoint in the average pain intensity score were also similar between the treatment groups (Fig. 1). The responder rate based on 30\% improvement in average pain score at study endpoint in the ITT data set was $30.8 \%$ for patients treated with fulranumab and $33.3 \%$ for placebo patients.

There were no statistically significant between-group differences for any of the secondary endpoints (Table 3),
Table 2 Demographic and baseline characteristics (ITT data set)

\begin{tabular}{|c|c|c|}
\hline Characteristic & $\begin{array}{l}\text { Placebo } \\
(N=17)\end{array}$ & $\begin{array}{l}\text { Fulranumab } 9 \mathrm{mg} \\
\qquad(N=14)\end{array}$ \\
\hline \multicolumn{3}{|l|}{ Sex, n (\%) } \\
\hline Female & $13(76.5)$ & $13(92.2)$ \\
\hline Male & $4(23.5)$ & $1(7.1)$ \\
\hline \multicolumn{3}{|l|}{ Race, n (\%) } \\
\hline White & $16(94.1)$ & $13(92.9)$ \\
\hline Black or African American & $1(5.9)$ & $1(7.1)$ \\
\hline \multicolumn{3}{|l|}{ Age (years) } \\
\hline Mean \pm SD & $46.2(13.56)$ & $50.6(10.68)$ \\
\hline Range & $25-69$ & $38-78$ \\
\hline \multicolumn{3}{|l|}{ Body weight (kg) } \\
\hline Mean \pm SD & $78.9(16.61)$ & $71.7(26.12)$ \\
\hline Range & $54-117$ & $46-148$ \\
\hline \multicolumn{3}{|c|}{ UPOINT clinical phenotyping, n (\%) } \\
\hline Urinary & $17(100)$ & $14(100)$ \\
\hline Psychosocial & $5(29)$ & $3(21)$ \\
\hline Organ-specific & $16(94)$ & $11(79)$ \\
\hline Infection & $4(24)$ & $5(36)$ \\
\hline Neurological/systemic & $7(41)$ & $9(64)$ \\
\hline Tender point & $12(71)$ & $9(64)$ \\
\hline \multicolumn{3}{|c|}{ Duration of interstitial cystitis (years) } \\
\hline Mean \pm SD & $9.4(9.87)$ & $5.8(5.44)$ \\
\hline Range & $2-40$ & $1-20$ \\
\hline \multicolumn{3}{|c|}{$\begin{array}{l}\text { Hunner's ulcer and/or glomerulation, n } \\
(\%)\end{array}$} \\
\hline Yes & $9(52.9)$ & $10(71.4)$ \\
\hline No & $8(47.1)$ & $4(28.6)$ \\
\hline
\end{tabular}

however, there was a trend of better GRA. Five (35.7\%) patients in the fulranumab group and 4 (23.5\%) patients in the placebo group reported a GRA score of "very much improved" or "much improved", 5 patients in each group ( $35.7 \%$ and $29.4 \%$, respectively) reported a GRA score of "minimally improved", and 4 (28.5\%) and 8 (47.1\%) patients in the respective groups reported "not changed", "minimally changed", or "much worse".

\section{Safety results}

The safety profile of fulranumab was similar to that of placebo. The most frequently reported adverse events in the fulranumab group were diarrhea, carpal tunnel syndrome, and urinary tract infection (Table 4). In both treatment groups, most adverse events were mild or moderate in intensity and were reported to be either not related or doubtfully related to the study drug. The only serious adverse event (kidney infection) was reported in the placebo group. 
Table 3 Summary of efficacy at end of double-blind treatment (ITT Data Set)

\begin{tabular}{|c|c|c|}
\hline & $\begin{array}{l}\text { Placebo } \\
(N=17)\end{array}$ & $\begin{array}{l}\text { Fulranumab } 9 \mathrm{mg} \\
\qquad(N=14)\end{array}$ \\
\hline \multicolumn{3}{|l|}{ Primary Endpoint - Average Pain Intensity Score ${ }^{a}$} \\
\hline Baseline, mean \pm SD & $6.8 \pm 1.30$ & $6.1 \pm 1.11$ \\
\hline Change at end of double-blind treatment period, mean \pm SD & $-1.2 \pm 1.88$ & $-1.4 \pm 1.41$ \\
\hline Difference of means (95\% Cl) & & $-0.2 \pm 0.64(-1.52,1.10)$ \\
\hline \multicolumn{3}{|l|}{ \% Responders defined as: } \\
\hline$\geq 30 \%$ improvement in average pain intensity score ${ }^{a}$ & $5(33.3 \%)$ & $4(30.8 \%)$ \\
\hline$\geq 50 \%$ improvement in average pain intensity score ${ }^{a}$ & $3(20.0 \%)$ & $3(23.1 \%)$ \\
\hline \multicolumn{3}{|l|}{ Secondary Endpoint - Worst Pain Intensity Score ${ }^{a}$} \\
\hline Baseline, mean \pm SD & $7.6 \pm 1.32$ & $6.9 \pm 1.13$ \\
\hline Change at end of double-blind treatment period, mean \pm SD & $-1.5 \pm 1.90$ & $-1.5 \pm 1.55$ \\
\hline Difference of means (95\% Cl) & & $-0.1 \pm 0.66(-1.44,1.28)$ \\
\hline \multicolumn{3}{|l|}{ Other Secondary Endpoints: } \\
\hline \multicolumn{3}{|l|}{ ICSI Total Score ${ }^{b}$} \\
\hline Baseline, mean \pm SD & $16.5 \pm 2.92$ & $14.6 \pm 3.27$ \\
\hline Change at end of double-blind treatment period, mean \pm SD & $-3.0 \pm 3.97$ & $-4.4 \pm 4.40$ \\
\hline Difference of means $(95 \% \mathrm{Cl})$ & & $-1.4 \pm 1.50(-4.50,1.65)$ \\
\hline \multicolumn{3}{|l|}{ PUF Total Score } \\
\hline Baseline, mean \pm SD & $26.2 \pm 6.86$ & $24.3 \pm 4.89$ \\
\hline Change at end of double-blind treatment period, mean \pm SD & $-4.2 \pm 5.38$ & $-6.1 \pm 5.16$ \\
\hline Difference of means (95\% Cl) & & $-1.9 \pm 1.91(-5.81,1.99)$ \\
\hline \multicolumn{3}{|l|}{ PPBC Score ${ }^{d}$} \\
\hline Baseline, mean \pm SD & $4.9 \pm 0.86$ & $4.7 \pm 0.61$ \\
\hline Change at end of double-blind treatment period, mean \pm SD & $-0.5 \pm 1.12$ & $-0.9 \pm 1.23$ \\
\hline Difference of means (95\% Cl) & & $-0.3 \pm 0.42(-1.19,0.54)$ \\
\hline \multicolumn{3}{|l|}{ Daytime urinary frequency ${ }^{e}$} \\
\hline Baseline, mean \pm SD & $16.5 \pm 11.66$ & $11.0 \pm 6.68$ \\
\hline Change at end of double-blind treatment period, mean \pm SD & $0.8 \pm 3.92$ & $0.5 \pm 2.95$ \\
\hline Difference of means $(95 \% \mathrm{Cl})$ & & $-0.3 \pm 1.33(-3.02,2.44)$ \\
\hline \multicolumn{3}{|l|}{ Nocturnal urinary frequency ${ }^{e}$} \\
\hline Baseline, mean \pm SD & $5.0 \pm 4.36$ & $3.8 \pm 3.28$ \\
\hline Change at end of double-blind treatment period, mean \pm SD & $0.6 \pm 5.33$ & $-0.4 \pm 1.33$ \\
\hline Difference of means $(95 \% \mathrm{Cl})$ & & $-1.0 \pm 1.52(-4.16,2.09)$ \\
\hline
\end{tabular}

ICSI O'Leary-Sant Interstitial Cystitis Symptoms Index, PPBC Patient Perception of Bladder Condition, PUF Pelvic Pain and Urgency/Frequency Questionnaire

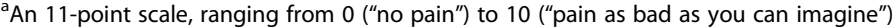

${ }^{\mathrm{b}} \mathrm{Sum}$ of 4 individual question ratings, each on a 0 to 5 scale, where higher scores indicate worse symptoms

${ }^{\mathrm{C}} \mathrm{A} 12$-item scale, with total score (sum of the symptom and bother subscale scores) ranging for 0 to 35 ; higher scores indicate worse symptoms

${ }^{\mathrm{d}}$ Single item global measure of bladder condition rated on a 6-point scale

eDerived as an average number of events over the 7 consecutive days prior to each visit

Mean of all non-missing scores after first dose and up to last non-missing score for the 12-week double-blind period

No study patient discontinued study drug due to an adverse event.

Regarding adverse events of clinical interest (related to anti-NGF antibodies), none of the study patients had joint-related serious adverse events. No event related to bradycardia, hepatic failure, acute renal failure, or neurologic and motor system was reported. One patient (in the placebo group) experienced a hypotension-related adverse event.

\section{Discussion}

In this multicenter, double-blind, randomized study of 31 adults with a diagnosis of IC/BPS who suffered from chronic, moderate-to-severe bladder pain, a treatment 


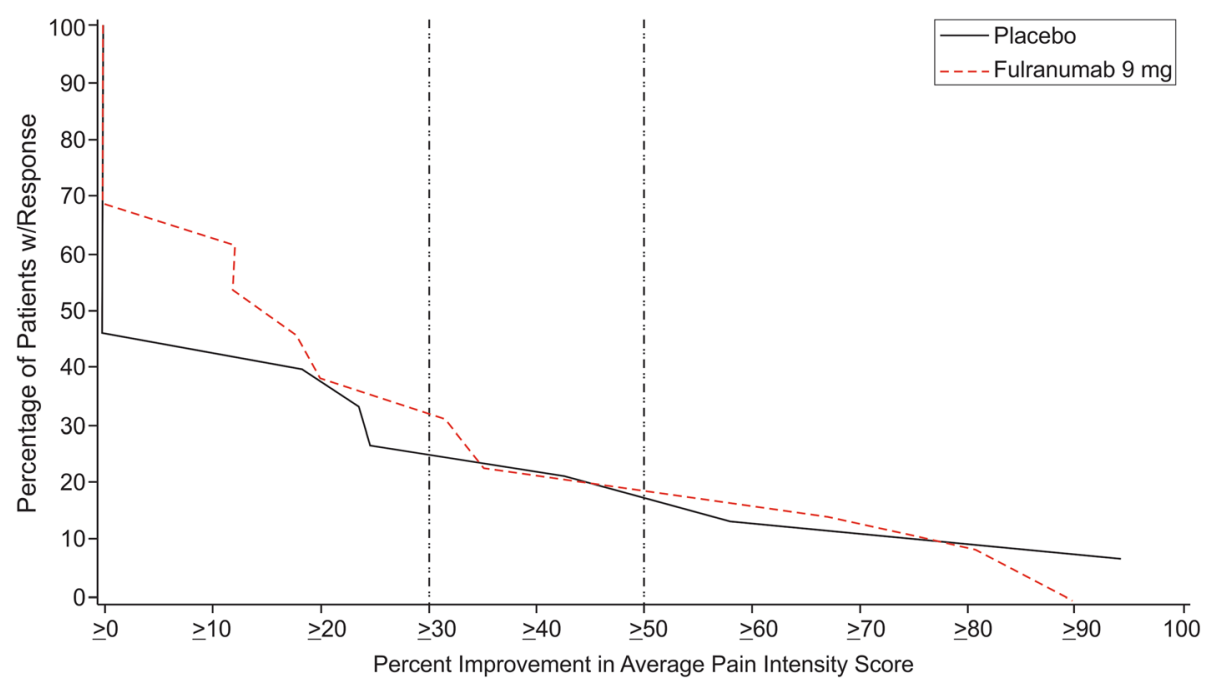

Fig. 1 Distribution of Pain Responder Rates at the Study Endpoint

effect (vs. placebo) was not observed with fulranumab, based on the primary study endpoint or any of the secondary endpoints. A trend for improvement was observed on the GRA, considering all subcategories, however this finding needs to be confirmed. Patient enrollment was curtailed at an early stage due to a regulatory hold on clinical studies, preventing complete efficacy and safety analysis, reducing power (from 79 to $52 \%$ ), and limiting conclusions. In addition, an imbalance in the baseline characteristics of the two treatment groups was detected, further diminishing the ability to definitively rule out a potential treatment effect. The development of anti-NGF antibodies was re-initiated in 2015 in patients with osteoarthritis and low back pain (NCT02301234, NCT0252818, NCT02528253, and NCT02683239; at clinical trials.gov). Further development of anti-NGF agents in patients with IC/BPS will be

Table 4 Treatment-emergent adverse events reported in $\geq 10 \%$ of patients in either treatment group

\begin{tabular}{lcc}
\hline & $\begin{array}{c}\text { Placebo } \\
(N=17)\end{array}$ & $\begin{array}{c}\text { Fulranumab 9 mg } \\
(N=14)\end{array}$ \\
\hline Any adverse event & $11(65 \%)$ & $8(57 \%)$ \\
Diarrhea & $2(12)$ & $3(21)$ \\
Carpal tunnel syndrome & 0 & $2(14)$ \\
Urinary tract infection & $2(12)$ & $2(14)$ \\
Pain & $2(12)$ & 0 \\
Pain in extremity & $2(12)$ & 0 \\
Paresthesia & $2(12)$ & 0 \\
Sinusitis & $2(12)$ & 0 \\
\hline
\end{tabular}

Adverse events are presented in decreasing order of incidence for the fulranumab group dependent on the safety profile observed in the osteoarthritis and low back pain studies.

The therapy of IC/PBS is usually multimodal and is rarely satisfactory [26-28]. With the exception of PPS (Elmiron), there are currently no approved pharmacologic therapies in the United States for IC/BPS. Many pharmacologic therapies such as antidepressants and antihistamines are used off label. Other therapies include intravesical installation of agents, bladder distention, and surgical intervention [26-28]. Promising agents have been studied, but with mixed results, and none have gained regulatory approval. In this regard, Evans et al. reported significant improvement in pain and the GRA 6 weeks after a single IV infusion of another anti-NGF monoclonal antibody, but no improvement on the ICSI in a pilot study of 64 patients with IC/BPS [14]. The same anti-NGF antibody was tested in a second clinical study of over 200 patients with IC/BPS that was terminated for futility (NCT00999518) [29].

Limitations of this study were early termination of the study resulting in small sample size and some patients not receiving 3 doses of study drug, as well as imbalance between the treatment groups based on baseline characteristics. For example, patients in the placebo group reported longer IC/BPS disease duration (mean, 9.4 vs. 5.8 years for the fulranumab group) and higher daytime urinary frequency (mean, 16.5 vs. 10.8); and of the UPOINT domains, the fulranumab group had a higher percentage of patients in the infection and neurological/ systemic domains than the placebo group (Table 2). In addition, due to the heterogeneous population, multiple factors that are impractical to stratify may confound or increase the noise of the study; these include concomitant IC/BPS medication types/rate of usage, and 
commonly comorbid conditions (e.g., irritable bowel disease, depression, pelvic floor dysfunctional disease) that define different clinical phenotypes [30].

Clinical trials of IC/BPS have been limited, in general, by substantial heterogeneity in methodology, symptoms assessment, duration of treatment, and follow-up [31]. For example, although initial trials were positive for PPS $[32,33]$, subsequent large confirmatory trials have had mixed results [34]. Experts have recently advanced the use of clinical phenotypes based on UPOINT or pain mapping/pain location in IC/BPS research [30,35]. With regard to analgesia for IC/BPS, future studies should include mapping of pain sites (i.e., restricted to the pelvis; pain sites beyond the pelvis) and the quality/severity of pain at those sites, assuring differentiation between IC/ BPS and other pain syndromes by patients, as well as correlate findings from these assessments with associated comorbid, chronic pain conditions [30]. While this study was designed to collect data pertaining to the different clinical phenotypes using the UPOINT system and conduct exploratory subgroup analyses, these analyses were not feasible due to early study termination and the reduced sample size. Future studies of adequate sample size should further explore treatment response according to clinical phenotypes.

\section{Conclusions}

In patients with moderate to severe chronic bladder pain from IC/BPS, fulranumab at the single dose $(9 \mathrm{mg})$ tested failed to show analgesic activity as compared with placebo, although the study findings are limited by early study termination and imbalance in the baseline characteristics of the study population. IC/BPS remains a difficult medical condition in need of satisfactory therapies.

\section{Abbreviations \\ ANOVA: Analysis of variance; FDA: Food and Drug Administration; GRA: Global response assessment; IC/BPS: Interstitial cystitis/bladder pain syndrome; ICSI: Interstitial cystitis symptom index; IT: Intent-to-treat; NGF: Nerve growth factor; NRS: Numerical rating scale; PPBC: Patient Perception of Bladder Condition; PPS: Pentosan polysulfate sodium; PUF: Pelvic Pain and Urgency/Frequency Questionnaire; SC: Subcutaneously; SD: Standard deviation}

\footnotetext{
Acknowledgments

Writing support was provided by Sandra Norris, Pharm.D. of the Norris Communications Group, LLC and funded by Janssen Research \& Development, LLC. The authors thank Luqiang Wang, PhD, contract statistician of Janssen, for review of and statistical contributions to the manuscript. Ellen Baum, Ph.D. (of Janssen Research \& Development, LLC) provided additional editorial assistance.

The authors also thank the study participants of this study, without whom the study would never have been accomplished, and the investigators for their participation in the study (i.e., randomized patients): ONTARIO CANADA Jack Barkin, MD; Joseph Zadra, MD; UNITED STATES: California: Edward Davis, MD; Bruce Khan, MD; Marianne Rochester, MD; Florida: Marc Gittelman, MD; Louisiana: Samuel Alexander, MD; Neil Baum, MD; Kevin Cline, MD; New Jersey: Eric Margolis, MD; New York: Evan Goldfischer, MD; and North Carolina Robert Lamar Parker, MD.
}

\section{Funding}

This study was funded by Janssen Research \& Development, LLC.

\section{Availability of data and materials}

The datasets used and/or analyzed during the current study are available from the corresponding author on reasonable request.

\section{Authors' contributions}

HW and JT were involved in study design and KMK and JT were involved in data review and analysis for the study. LJR served as the Medical Monitor for the study and reviewed data and analyses for the study. SW was involved in study design, data review and analysis, and performed the statistical analysis. All authors contributed to writing drafts of the article. All authors approved the final version of the article, including the authorship list.

\section{Competing interests}

Dr. Hao Wang was an employee of Janssen Research \& Development, LLC, in Raritan/Titusville, NJ, USA during the conduct of this study; Dr. Wang currently declares no other conflicts of interest.

Drs. Kathleen Kelly, Steven Wang, and John Thipphawong are employees of Janssen Research \& Development, LLC, in Raritan/Titusville, NJ, USA. Dr. Lucille Russell was an employee of Janssen Research \& Development, LLC, in Raritan/Titusville, NJ, USA at the time this study was conducted and this manuscript was written and submitted for publication.

\section{Consent for publication}

This manuscript does not include details, images, or videos relating to individual participants, therefore there was no need to obtain written informed consent for publication from the participants.

\section{Ethics approval and consent to participate}

The study protocol and its amendments were approved by an Independent Review Board or Research Ethics Board at the following sites: Canada - The Male Health Centre, Toronto; and, the Male/Female Health and Research Centre, Barrie, Ontario; United States - California: Citrus Valley Medical Research, Glendora; San Diego Uro-Research, San Diego; Scripps Clinic, San Diego; Florida: South Florida Medical Research, Aventura; Louisianna: Clinical Trials Management, LLC, Metairie; Best Clinical Trials, New Orleans; Regional Urology, Shreveport; New Jersey: Urology Center of Englewood, Englewood; New York: Hudson Valley Research, Poughkeepsie; North Carolina: Lyndhurst Clinical Research, Winston Salem.

\section{Author details}

${ }^{1}$ Office of Translational Research, National Institute of Neurological Disorders and Stroke (NINDS), Bethesda, MD, USA. ${ }^{2}$ Janssen Research \& Development, LLC, Raritan/Titusville, NJ, USA.

Received: 10 November 2015 Accepted: 14 December 2016 Published online: 05 January 2017

\section{References}

1. Hanno PM, Erickson D, Moldwin R, Faraday MM. Diagnosis and treatment of interstitial cystitis/bladder pain syndrome: AUA Guideline Amendment. J Urol. 2015;193(5):1545-53.

2. Clemens JQ, Link CL, Eggers PW, Kusek JW, Nyberg Jr LM, McKinlay JB, BACH Survey Investigators. Prevalence of painful bladder symptoms and effect on quality of life in black, Hispanic and white men and women. J Urol. 2007;177(4):1390-4.

3. Rothrock N, Lutgendorf SK, Kreder KJ. Coping strategies in patients with interstitial cystitis: relationships with quality of life and depression. J Urol. 2003;169(1):233-6.

4. Warren JW, Howard FM, Cross RK, Good JL, Weissman MM, Wesselmann U, Langenberg P, Greenberg P, Clauw DJ. Antecedent nonbladder syndromes in case-control study of interstitial cystitis/painful bladder syndrome. Urology. 2009;73(1):52-7.

5. Wesselmann U. Interstitial cystitis: a chronic visceral pain syndrome. Urology. 2001;57(6 Suppl 1):32-9.

6. Chong $M$, Hester J. Pharmacotherapy for neuropathic pain with special reference to urogential pain. In: Baranowski A, Abrams P, Fall M, editors. Urogenital pain in clinical practice. New York: Informa Healthcare USA, Inc; 2007. p. 427. 
7. Hefti FF, Rosenthal A, Walicke PA, Wyatt S, Vergara G, Shelton DL, Davies AM. Novel class of pain drugs based on antagonism of NGF. Trends Pharmacol Sci. 2006;27(2):85-91.

8. Ochodnicky P, Cruz CD, Yoshimura N, Michel MC. Nerve growth factor in bladder dysfunction: contributing factor, biomarker, and therapeutic target. Neurourol Urodyn. 2011;30(7):1227-41.

9. Fujita M, Kasai E, Omachi S, Sakaguchi G, Shinohara S. A novel method for assessing bladder-related pain reveals the involvement of nerve growth factor in pain associated with cyclophosphamide-induced chronic cystitis in mice. Eur J Pain. 2015. doi: 10.1002/ejp.693. [Epub ahead of print].

10. Schnitzer TJ, Marks JA. A systematic review of the efficacy and general safety of antibodies to NGF in the treatment of OA of the hip or knee. Osteoarthritis Cartilage. 2015;23 Suppl 1:S8-17.

11. Wang H, Romano G, Frustaci ME, Bohidar N, Ma H, Sanga P, Ness S, Russell $\sqcup$, Fedgchin M, Kelly KM, Thipphawong J. Fulranumab for treatment of diabetic peripheral neuropathic pain: a randomized controlled trial. Neurology. 2014;83(7):628-37.

12. Bramson C, Herrmann DN, Carey W, Keller D, Brown MT, West CR, Verburg $\mathrm{KM}$, Dyck PJ. Exploring the role of tanezumab as a novel treatment for the relief of neuropathic pain. Pain Med. 2015;16(6):1163-76.

13. Leite VF, Buehler AM, El Abd O, Benyamin RM, Pimentel DC, Chen J, Hsing WT, Mazloomdoost D, Amadera JE. Anti-nerve growth factor in the treatment of low back pain and radiculopathy: a systematic review and a meta-analysis. Pain Physician. 2014;17(1):E45-60.

14. Evans RJ, Moldwin RM, Cossons N, Darekar A, Mills IW, Scholfield D. Proof of concept trial of tanezumab for the treatment of symptoms associated with interstitial cystitis. J Urol. 2011;185(5):1716-21.

15. Nickel JC, Atkinson G, Krieger JN, Mills IW, Pontari M, Shoskes DA, Crook TJ. Preliminary assessment of safety and efficacy in proof-of-concept, randomized clinical trial of tanezumab for chronic prostatitis/chronic pelvic pain syndrome. Urology. 2012;80(5):1105-10.

16. Sanga P, Katz N, Polverejan E, Wang S, Kelly KM, Haeussler J, Thipphawong J. Efficacy, safety, and tolerability of fulranumab, an anti-nerve growth factor antibody, in the treatment of patients with moderate to severe osteoarthritis pain. Pain. 2013;154(10):1910-9.

17. Mayorga A, Wang S, Wang S, Kelly K, Thipphawong J. Double-blind, randomized study to evaluate efficacy and safety of fulranumab in patients with moderate to severe, chronic knee pain from osteoarthritis: interim analysis results (abstract 375). J Pain. 2013;14(4):S69.

18. Sanga P, Karcher K, Wang S, Kelly K, Oh C, Thipphawong J. Efficacy, safety, and tolerability of fulranumab in treatment of patients with moderate-tosevere, chronic low back pain (abstract 310). J Pain. 2011;12(4):53.

19. Hochberg MC. Serious joint-related adverse events in randomized controlled trials of anti-nerve growth factor monoclonal antibodies. Osteoarthritis Cartilage. 2015;23 Suppl 1:S18-21.

20. O'Leary MP, Sant GR, Fowler Jr FJ, Whitmore KE, Spolarich-Kroll J. The interstitial cystitis symptom index and problem index. Urology. 1997; 49(Suppl 5A):58-63.

21. Lubeck DP, Whitmore K, Sant GR, Alvarez-Horine S, Lai C. Psychometric validation of the O'leary-Sant interstitial cystitis symptom index in a clinical trial of pentosan polysulfate sodium. Urology. 2001;57(6 Suppl 1):62-6.

22. Dworkin RH, Turk DC, Wyrwich KW, Beaton D, Cleeland CS, Farrar JT, Haythornthwaite JA, Jensen MP, Kerns RD, Ader DN, Brandenburg N, Burke LB, Cella D, Chandler J, Cowan P, Dimitrova R, Dionne R, Hertz S, Jadad AR, Katz NP, Kehlet H, Kramer LD, Manning DC, McCormick C, McDermott MP, McQuay HJ, Patel S, Porter L, Quessy S, Rappaport BA, Rauschkolb C, Revicki DA, Rothman M, Schmader KE, Stacey BR, Stauffer JW, von Stein T, White RE, Witter J, Zavisic S. Interpreting the clinical importance of treatment outcomes in chronic pain clinical trials: IMMPACT recommendations. J Pain. 2008;9(2):105-21.

23. Warren JW, Meyer WA, Greenberg P, Horne L, Diggs C, Tracy JK. Using the International Continence Society's definition of painful bladder syndrome. Urology. 2006;67(6):1138-42.

24. Coyne KS, Matza LS, Kopp Z, Abrams P. The validation of the patient perception of bladder condition (PPBC): a single-item global measure for patients with overactive bladder. Eur Urol. 2006;49:1079-86.

25. Parsons $C L$, Dell J, Stanford EJ, Bullen M, Kahn BS, Waxell T, Koziol JA. Increased prevalence of interstitial cystitis: previously unrecognized urologic and gynecologic cases identified using a new symptom questionnaire and intravesical potassium sensitivity. Urology. 2002;60(4):573-8.
26. Rourke W, Khan SA, Ahmed K, Masood S, Dasgupta P, Khan MS. Painful bladder syndrome/interstitial cystitis: aetiology, evaluation and management. Arch Ital Urol Androl. 2014;86(2):126-31.

27. Davis NF, Brady CM, Creagh T. Interstitial cystitis/painful bladder syndrome: epidemiology, pathophysiology and evidence-based treatment options. Eur J Obstet Gynecol Reprod Biol. 2014;175:30-7.

28. Dyer AJ, Twiss CO. Painful bladder syndrome: an update and review of current management strategies. Curr Urol Rep. 2014;15(2):384.

29. Pfizer. Tanezumab Arthritis Advisory Briefing Document, 8 February 2012. Available at: http://www.fda.gov/downloads/AdvisoryCommittees/ CommitteesMeetingMaterials/Drugs/ArthritisAdvisoryCommittee/ UCM295205.pdf. Accessed 4 November 2015.

30. Nickel JC, Tripp DA, International Interstitial Cystitis Study Group. Clinical and psychological parameters associated with pain pattern phenotypes in women with interstitial cystitis/bladder pain syndrome. J Urol. 2015;193(1):138-44.

31. Giannantoni A, Bini V, Dmochowski R, Hanno P, Nickel JC, Proietti S, Wyndaele JJ. Contemporary management of the painful bladder: a systematic review. Eur Urol. 2012;61(1):29-53.

32. Sant GR, Propert KJ, Hanno PM, Burks D, Culkin D, Diokno AC, Hardy C, Landis JR, Mayer R, Madigan R, Messing EM, Peters K, Theoharides TC, Warren J, Wein AJ, Steers W, Kusek JW, Nyberg LM, Interstitial Cystitis Clinical Trials Group. A pilot clinical trial of oral pentosan polysulfate and oral hydroxyzine in patients with interstitial cystitis. J Urol. 2003;170(3):810-5.

33. Parsons CL, Benson G, Childs SJ, Hanno P, Sant GR, Webster G. A quantitatively controlled method to study prospectively interstitial cystitis and demonstrate the efficacy of pentosan polysulfate. J Urol. 1993:150(3):845-8.

34. Nickel JC, Forrest J, Barkin J, Payne C, Mosbaugh P. Safety and efficacy of up to $900 \mathrm{mg} /$ day polysulfate sodium (Elmiron) in patients with interstitial cystitis. Urology. 2001;57(6 suppl 1):122-3.

35. Nickel JC, Irvine-Bird K, Jianbo L, Shoskes DA. Phenotype-directed management of interstitial cystitis/bladder pain syndrome. Urology. 2014;84(1):175-9.

\section{Submit your next manuscript to BioMed Central and we will help you at every step:}

- We accept pre-submission inquiries

- Our selector tool helps you to find the most relevant journal

- We provide round the clock customer support

- Convenient online submission

- Thorough peer review

- Inclusion in PubMed and all major indexing services

- Maximum visibility for your research

Submit your manuscript at www.biomedcentral.com/submit
) Biomed Central 